\title{
FISCALIDAD IBEROAMERICANA, SIGLOS XVII-XX. TRANSICIONES, DISEÑOS ADMINISTRATIVOS Y JURÍDICOS
}

\author{
Carlos Alfonso Díaz Martínez \\ El Colegio de México, México
}

https://doi.org/10.36105/iut.2021n33.14

Luis Jáuregui y Carlos de Jesús Becerril Hernández (eds.), Fiscalidad iberoamericana, siglos XVII-XX. Transiciones, diseños administrativos y jurídicos, México, Instituto de Investigaciones Dr. José María Luis Mora, Universidad Anáhuac México, 2018, 335 pp. ISBN: 978-607-8611-18-8 (Instituto Mora), 978-607-8566-37-2 (Universidad Anáhuac México).

El libro coordinado por Luis Jáuregui y Carlos Becerril, resultado de las presentaciones en una mesa del Quinto Congreso Latinoamericano de Historia Económica (CLADHE V), articula diez estudios de caso sobre Nueva España y México (5), Brasil (3), Buenos Aires (1), y Ecuador (1), que abarcan el periodo colonial (2), la transición de órdenes soberanos (2), y la era independiente (6), más un estudio historiográfico de los editores que da apertura al volumen. Para ser justo con los aportes que ha hecho cada autor y tratar de darle un hilo conductor a la diversidad de preocupaciones que están plasmadas en los capítulos, he decidido presentar los puntos que en mi opinión distinguen cada contribución, y se relacionan con la introducción de los coordinadores del libro. Aunque, debo admitirlo, no fue tarea fácil reseñar esta obra.

La reflexión historiográfica de Jáuregui y Becerril cumple dos propósitos: explicar la utilidad de los estudios de historia fiscal, y reconstruir las varias tradiciones que han contribuido al examen del pasado tributario latinoamericano. En el primer caso, los editores sostienen que la historia permite conocer las trayectorias que conformaron la crítica situación fiscal de nuestros días, que está signada por una marcada desigualdad en la imposición, servilismo legislativo, evolución dependiente de los flujos externos de capitales y mercancías, baja carga impositiva, resistencias de ciudadanos acaudalados y poderosos, limitado control sobre 
el Erario, gasto público variable, entre otros seculares y comunes problemas que podrían deberse a la naturaleza de los sistemas fiscales "latinos": se gravan más las actividades económicas que las personas. Dicho en sus propios términos: "para comprender la situación fiscal actual de América Latina es necesario conocer los hitos y las experiencias de diversos ámbitos geográficos" (p. 8).

En el segundo caso, Jáuregui y Becerril trazan una genealogía de los estudios históricos fiscales, que incluye autores destacados como Marcello Carmagnani, Juan Carlos Garavaglia, Herbert Klein, Carlos Marichal, Luis Aboites, Angelo Carrera, por solo mencionar algunos investigadores de una larga y diversa lista. De este repaso los autores concluyen que la historiografía fiscal latinoamericana ha hecho énfasis en las herencias coloniales de los sistemas tributarios nacionales, la limitada transición hacia la modernidad fiscal, y las precarias reformas generales a la organización hacendaria. Asimismo, Jáuregui y Becerril constatan cierto desequilibrio en el desarrollo de los estudios históricos fiscales de los países latinoamericanos, pues si bien México y Argentina marcan la parada, países como Chile o Costa Rica tienen muy pocos trabajos sobre su pasado tributario. Este punto, me parece, es un reflejo del anterior, pues el progreso de las ciencias sociales en general, y de la historia en particular, depende de un sistema de incentivos ofrecidos por el Estado, a nivel educativo y financiero. Veamos de qué manera los demás capítulos atendieron las preocupaciones enunciadas por los coordinadores del libro.

Martín Wasserman y Bruno Aidar ofrecen dos estudios de caso sobre los sistemas tributarios de Buenos Aires y San Pablo durante el periodo colonial, respectivamente. Mientras Wasserman estudia la conformación del orden colonial, Aidar examina su reforma y ocaso. En este sentido, Wasserman está interesado por escudriñar las transformaciones institucionales que revirtieron la dirección de las transferencias fiscales entre Potosí y Buenos Aires, o de cómo la ciudad porteña pasó de remitente a receptora de caudales. Para este autor el cambio de sentido de las remesas reflejó una modificación de la manera de entender la seguridad portuaria rioplatense, que privilegió la defensa antes que la protección. Es decir, si en el primer caso era más seguro trasladar dinero a Potosí que conservarlo en Buenos Aires, en el segundo el resguardo de la plaza requería enfrentar la amenaza militar, a fin de defender no solo las cantidades almacenadas en la caja real sino también el sistema portuario y los circuitos legales y semilegales que sostenían la vida económica y financiera porteña. Asimismo, esta mutación en la organización defensiva de Buenos Aires fue impulsada por los empleados del Erario regio interesados en garantizar la autonomía financiera de la hacienda local, así como ofrecer amparo a los mercaderes ultramarinos y minoristas de la ciudad, muchos de los cuales eran sus fiadores para el ejercicio del cargo. De esta manera, se vinculan ambos procesos. Un creciente tráfico mercantil hizo necesario erigir armadas, batallones y presidios para cuidar el flujo regular de caudales del comercio a las cajas reales, y fue posible gracias a la colusión de intereses entre comerciantes y empleados hacendarios.

Bruno Aidar también estudia la imbricación entre funcionarios del Erario y grupos de interés, pero el foco es la capitanía general de San Pablo, entre finales 
del siglo XVIII y comienzos del siglo XIX. El interés del autor es indagar los mecanismos de negociación entre corporaciones civiles y económicas con las oficinas del fisco local a fin de obtener la aprobación real para el establecimiento de contribuciones, o ganar subastas de arrendamiento de algunas rentas del Erario. Para lograr estos cometidos, azucareros, hacendados y funcionarios nombraban procuradores que debían pactar con los ministros metropolitanos. Estos arreglos eran importantes porque después de las reformas pombalinas, el equivalente portugués del reformismo borbónico español, el poder corporativo fue reducido a expensas del poder real, por lo que la Hacienda portuguesa recuperó rentas cedidas a los vasallos o monopolizó la facultad de dictar contribuciones. Esto generó competencia entre los mercaderes brasileros y los gremios mercantiles de Lisboa por el arriendo de impuestos. Igualmente, porque la capitanía brasilera requería autonomía financiera para generar recursos que cubrieran los crecientes gastos defensivos, pese a que San Pablo recibía una transferencia regular de Río de Janeiro.

Pese a que los estudios de Wasserman y Aidar tratan sobre territorios y periodos distintos, sus investigaciones reflejan coincidencias en la organización institucional de la fiscalidad española y portuguesa. Además de la normativa regia y local, el desempeño del fisco requirió del engarce entre funcionarios y empresarios, y de mecanismos legales e ilícitos, pues si los rendimientos del Erario dependían de la situación del comercio transatlántico y del apoyo de los acaudalados empresarios para el pago de fianzas y socorros en situaciones extraordinarias, los grupos mercantiles necesitaban de protección, certidumbre o simple omisión para la realización de sus actividades comerciales. Empero, los autores no proporcionan mucha información acerca del tipo de vínculos que unían a comerciantes y empleados del Estado, ni especifican el grado de cohesión de las alianzas que mantenían en operación los negocios privados y públicos. Indicar si estos enlaces se tendieron entre familia, paisanos, compadres o amigos permitiría captar la fortaleza del lazo social, tal y como ha sido estudiado por Guillermina del Valle en varias investigaciones.

La transición entre órdenes fiscales coloniales e independientes en México y Brasil es estudiada por Ernest Sánchez Santiró y Eduardo Silva, respectivamente. Sánchez Santiró analizó las modificaciones en el cuadro de rentas de Nueva España/México entre finales del siglo XVIII y el fin de la república federal. De acuerdo con el autor, la mutación solo es entendible si se tienen en cuenta las particularidades del turbulento periodo 1808-1821: deterioro general de las rentas, introducción del comercio libre, derogación impositiva, pérdida de control sobre el Erario, flexibilización en la administración de los estancos, repunte de las contribuciones indirectas, e irrupción de los impuestos directos y progresivos. También, según el autor, es importante reconocer que el cambio fue lento y bastante continuista, pues deliberadamente se evitaron las rupturas. En este sentido, Sánchez Santiró es bastante categórico al afirmar que el pacto federal no creó un nuevo sistema de impuestos, porque lo que solamente sucedió fue un reparto de las rentas que conformaban el Erario Regio entre la federación y las entidades federativas. La única novedad establecida fue el "contingente": una contribución 
de los Estados a la unión. Dada esta segmentación del conjunto de ingresos de la Hacienda, la federación dependió de los impuestos al comercio exterior, hasta casi el último cuarto del siglo XIX.

Eduardo Silva investiga la creación del sistema fiscal nacional de Brasil, que fue un proceso lleno de dificultades del reinado de Juan VI al imperio de Pedro I. De acuerdo con el autor, la corte portuguesa heredó una política ineficiente para cubrir los gastos del Estado, pues la posición comercial de Gran Bretaña como nación más favorecida minó los ingresos, inhibió el desarrollo del Banco de Brasil, y limitó el desarrollo de las contribuciones internas. Por ejemplo, en aras de eliminar la revolución pernambucana de 1817, el gobierno brasilero recurrió a emisiones monetarias y préstamos contratados en el extranjero, dada la estrechez del Erario. Para revertir esta tendencia y reformular los mecanismos de recaudo de impuestos y gestión del gasto, las administraciones de la Regencia y el Imperio implementaron varias instituciones. En este sentido, en 1829 fue promulgado el primer reglamento general para el funcionamiento de la Tesorería Nacional y las Juntas de Hacienda, a fin de organizar el presupuesto del Estado e incrementar el poder del ejecutivo sobre el fisco. Por otra parte, dado que la fiscalidad se concentró en el comercio exterior fue necesario simplificar su administración para agilizar el comercio y reducir los fraudes. Así, los esfuerzos por corregir y actualizar la dirección tributaria del país se centraron en las aduanas, y llevaron a la proclamación del primer reglamento general en 1832. Por último, la búsqueda de un mejor manejo de las rentas del café, azúcar, aguardiente y comercio de esclavos condujo a la formación de la Administración de Diversas Rentas Nacionales (1833), que tuvo oficinas en las provincias brasileñas, y que en 1836 se fusionó con la dirección de aduanas.

Los estudios de Sánchez Santiró y Silva demuestran la importancia de los años revolucionarios para la conformación de los erarios nacionales, las dificultades que enfrentaron los primeros gobiernos independientes para restaurar el erosionado control sobre el fisco, definir las prerrogativas tributarias de las provincias y la nación, así como la necesidad que tuvieron los gobiernos de formular instituciones que graduaran las transformaciones del sistema y garantizaran cierta estabilidad financiera y política.

Luis Jáuregui dedicó su capítulo a la transición entre la primera república federal y la república centralista mexicanas. Para el autor, el sistema federativo mexicano sufrió de "fallas de origen", debido a que naufragó en la insostenibilidad fiscal ocasionada por la abolición tributaria de Iturbide (1821) y el reparto de 1824. Luego vinieron tímidas implementaciones de nuevas contribuciones, reformas administrativas y contables. Así, debido carencia de información y la falta de control, la federación solo contó con capacidad para gravar a los Estados, y basó su relación fiscal con las entidades federativas sobre la renta del tabaco y el contingente. La fragilidad fiscal del sistema federal fue agravada por la abolición del estanco de tabaco, pese a que se impuso el derecho de alcabala sobre la venta de tabaco. Para modificar esta trayectoria, en 1832 se dictó una reforma que entregó el control de la contabilidad estatal a la federación, con lo cual se despojó a los 
Estados parte de su soberanía. Fue la antesala de la república centralista: la conclusión casi que inevitable de un sistema federal poco reflexionado, y sujeto al poder de las poderosas y acaudalas élites estatales.

Marcia Eckert estudia la formación de una entidad estatal que nos recuerda que las fronteras de los Estados están sujetas a cuestionamientos y revisiones: del reto secesionista de la provincia de Río Grande de San Pedro (1835) surgió el Estado Rio-Grandense (1836-1845). Como toda nueva formación estatal, la experiencia Riograndense debió enfrentar los problemas y dilemas que supone darle una organización administrativa al Estado, definir ingresos estables, y ordenar el sistema monetario. Para alcanzar estos objetivos, se formularon varias políticas gubernamentales como la reestructuración administrativa y tributaria (1838), y la amortización de la moneda falsa que circulaba en el Estado. Con el fin de amortizar las monedas se asignaron fondos provenientes de los ingresos aduaneros, donativos, préstamos, y el embargo de bienes de los enemigos políticos. En suma, un cuadro de rentas típico de todo periodo revolucionario. Empero, el recaudo fue insuficiente, y el gobierno se vio obligado a recurrir a la confiscación para pagar a las tropas. En última instancia, el Estado Rio-Grandense desapareció.

Javier Torres Medina analiza los vaivenes de la capitación de indios en México durante la primera mitad del siglo XIX. De un impuesto que representaba el derecho eminente del monarca sobre los territorios americanos, el tributo se convirtió en "contribución personal" durante la primera república federal, y pasó a ser una capitación en la república centralista vinculada a la mera existencia civil de las personas (1838). De acuerdo con el autor, este gravamen no fue equitativo ni igualitario, nunca se buscó su progresividad, y su rechazo fue generalizado. Sin embargo, dado que sus productos se destinarían al pago del ejército, el presidente puso el mayor interés en la recaudación. Para ello se nombraron comisionados que debían formar censos poblacionales a fin de definir la cuota de cada causante. Debido al descontento que generó su cobro, la resistencia de los afectados no se hizo esperar, y se expresó en la presentación de documentos falsos para las liquidaciones, así como en protestas y motines, especialmente de comunidades indígenas. Según Torres Medina, los contribuyentes no veían en el Estado un proveedor de bienes públicos, sino un expoliador de recursos privados. Además, los indígenas reconocieron la injusticia de estar obligados a pagar impuestos, aunque no eran ciudadanos con plenos derechos. En suma, fue un proyecto fallido incentivado por la urgencia financiera, que a la larga impidió la consolidación de un sistema fiscal liberal basado en contribuciones directas y progresivas.

Luis Jáuregui, Marcia Eckert y Javier Torres Medina plantean las encrucijadas que enfrentaron los nacientes Estados mexicano y riograndense a fin de consolidar la soberanía fiscal y modernizar su estructura de rentas, porque sin importar los esfuerzos, este proceso fue limitado por la debilidad propia de los regímenes después de la independencia, y la incapacidad de los gobiernos nacionales para derrotar la resistencia de los territorios que los constituían. Empero, como demuestran los autores de los siguientes capítulos, en periodos posteriores la construcción estatal también debió enfrentar problemas similares. 
Carlos Becerril indaga la justicia fiscal entre la quiebra del porfiriato y la consolidación del gobierno posrevolucionario mexicano. Su tesis es que la revolución mexicana (1910-1917) no modificó el proyecto fiscal contenido en el juicio de amparo, creado a mediados del siglo anterior. Es decir, la organización institucional del porfiriato en materia fiscal, caracterizada por la definición y protección de los derechos de propiedad, fue conservada después del proceso revolucionario, pero fue encaminada hacia otros objetivos de gobierno acordes con el programa político de la facción vencedora al final de la guerra civil. De acuerdo con Becerril, aunque el sistema jurídico mexicano dejó de tener vigencia con la caída de Francisco I. Madero en 1913, y con ello el amparo quedó en suspenso, la promulgación de la constitución de 1917 restauró su vigencia, pues en el texto constitucional están plasmadas las garantías que el juicio de amparo protege en materia fiscal: que las contribuciones sean proporcionales, equitativas y se apeguen al debido proceso. En conclusión, pese a la retórica revolucionaria sobre la creación de un orden totalmente nuevo sobre las "ruinas" del antiguo régimen porfirista, hay un nexo de continuidad entre las constituciones de 1857 y 1917 que los revolucionarios triunfantes decidieron mantener.

María del Ángel Molina reflexionó sobre el proceso de centralización fiscal del Erario mexicano en el siglo xx. La estrategia del gobierno federal fue el proyecto de "coordinación" que unificaba el sistema tributario de la federación y las entidades federativas. El instrumento fue el Impuesto Sobre Ingresos Mercantiles (ISIM), que reemplazaría el derecho federal sobre el timbre. Su implementación tomó varios años (1947-1973) debido a la resistencia de los Estados. La táctica fue la celebración de convenios particulares entre las entidades federativas y la federación, aunque no fue el único método ensayado, pues como indica la autora, desde 1925 la unión formuló planes que fracasaron por el freno de los Estados, como una reforma constitucional o la proclamación de una ley general. A fin de lograr la centralización, el gobierno federal obvió el espinoso asunto de la concentración de prerrogativas fiscales, por medio de la cual conculcaba la soberanía tributaria de las entidades federativas, y centró la discusión en la distribución de los productos de las rentas. Aunque el objetivo declarado de la "coordinación" era eliminar la competencia fiscal, nos dice la autora, se produjo precisamente esto, pues la federación y los Estados se enfrentaron por las atribuciones tributarias de cada nivel de gobierno. En suma, este capítulo nos muestra cómo la resistencia de diversos actores altera la política fiscal, y de qué manera el Estado es lo suficientemente flexible para modificar las tácticas para implementar una misma estrategia.

El último capítulo del libro está dedicado al estudio de la estructura y evolución de los ingresos y gastos del Estado ecuatoriano durante el siglo Xx. Sus autores, Atenea Castillo y Reto Bertoni, reconstruyeron series de ambas variables presupuestales, y explicaron los cambios en su nivel y composición por los arreglos institucionales entre entidades exactoras y causantes. En este sentido, en concordancia con el típico librecambismo decimonónico, a principios del siglo XX los impuestos aduaneros dominaban los ingresos del gobierno de Ecuador. Hacia 
1950, el cuadro de rentas era un poco más equilibrado: dominaban los impuestos aduaneros, y seguían en importancia los indirectos internos, mientras que la participación de los directos rodeaba el 10\%. A finales de la centuria la renta petrolera abarcaba la mayor parte de las entradas tributarias del Estado ecuatoriano, y era complementada por los impuestos indirectos internos, los directos y los derechos aduaneros. En otras palabras, un siglo después la nación ecuatoriana reemplazó la dependencia de las fluctuaciones del comercio exterior por la subordinación a los vaivenes de las regalías, ventas de la empresa pública de hidrocarburos, e impuestos a las corporaciones privadas petroleras. Según Castillo y Bertoni, la tímida modernización fiscal de mediados de siglo se estancó, pues se frenó el aumento absoluto y relativo de la tributación directa. Parece que, dos siglos después de la independencia, América Latina sigue esperando el establecimiento de sistemas fiscales modernos y liberales.

Para concluir esta reseña, quisiera subrayar algunos puntos que a mi parecer revelan un cambio historiográfico respecto a los estudios de historia fiscal previos. En primer lugar, con excepción del texto de Atenea Castillo y Reto Bertoni que llena un vacío cuantitativo importante, los autores del presente volumen se han ocupado de investigar las reglas de juego que definen la organización y gobierno de los sistemas fiscales, en contraste con las clásicas investigaciones que se esmeraron por elaborar series presupuestales. En segundo lugar, también el rango de visión se ha ampliado, pues si antes la atención recaía sobre las entidades exactoras, ahora se ha desplazado hacia los contribuyentes y sus vínculos con los funcionarios de gobierno. En tercer lugar, el comportamiento del recaudo ya no se entiende solamente como un efecto de la presión fiscal o el desempeño de la economía, sino como resultado del grado de control que ejerce el fisco, así como de la colaboración y resistencia de los causantes respecto del Estado. Finalmente, ha surgido el interés por examinar el proceso fiscal que existe entre la elaboración de la ley y la rendición de cuentas de ejecución presupuestal, y que pasa por el diseño de reglamentos administrativos, teneduría de documentos contables, vigilancia de las actividades económicas de los contribuyentes, elección y formación de los empleados fiscales, entre otros procedimientos por los cuales un gobierno redistribuye ingresos de quienes pagan impuestos hacia quienes reciben pagos del Estado.

Esta obra está bajo licencia internacional Creative Commons ReconocimientoNo-Comercial-CompartirIgual 4.0.

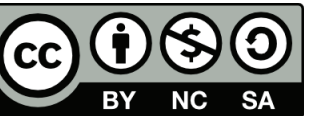

